\title{
Structure and function of the respiratory muscles in patients with COPD: impairment or adaptation?
}

\author{
M. Orozco-Levi
}

Structure and function of the respiratory muscles in patients with COPD: impairment or adaptation?. M. Orozco-Levi. (C) ERS Journals Ltd 2003.

ABSTRACT: Respiratory muscles are essential to alveolar ventilation. These muscles work against increased mechanical loads due to airflow limitation and geometrical changes of the thorax derived from pulmonary hyperinflation. Respiratory muscle fibres show several degrees of impairment in cellular and subcellular structures which, in many cases, are proportional to the severity of the disease and accompanying conditions (ageing, deconditioning, starvation, comorbidity). This structural impairment translates, from the functional point of view, to a loss of strength (capacity to generate tension) and an increased susceptibility to failure in the face of a particular load (early onset of fatigue).

On the other hand, there is accumulating evidence that the diaphragm and other respiratory muscles are also able to express adaptive changes in response to the chronic mechanical load imposed by the disease. In most cases, impairment and adaptation of the respiratory muscles reaches a balance that permits enough ventilation for patients' survival. However, this balance can be altered for additional increments of the mechanical or metabolic load on the muscles (e.g. abdominal or thoracic surgeries, pneumonia, pulmonary embolism, etc.). Moreover, loss of balance is not always associated with extreme situations. Many patients develop ventilatory failure and require hospital admission even if the cause of the exacerbation is less dramatic (bronchial infections, pain of any nature, electrolyte disturbances, etc.).

Although the physiopathology of chronic obstructive pulmonary disease exacerbations is multifactorial, the above-mentioned fragility suggests the existence of a "fragile balance" between respiratory muscle overload and respiratory muscle adaptations. Assessment of respiratory muscle function through specific tests evaluating the strength and endurance could offer valuable information about this particular susceptibility to muscle imbalance. Identification of patients possessing a fragile respiratory muscle balance could have important implications for the application of specific strategies such as respiratory muscle training, nutrition, or anabolic treatment.

Eur Respir J 2003; 22: Suppl. $4641 s-51 s$.
Correspondence: M. Orozco-Levi

Muscle Research Unit

Servei de Pneumologia

Hospital del Mar

Institut Municipal

d'Investigació Mèdica (IMIM)

CEXS-Universitat

Pompeu Fabra

Carrer Dr. Aiguader, 80

Barcelona

E-08003 Spain.

Fax: 34932213237

E-mail: morozco@imim.es

Keywords: Adaptation

chronic obstructive pulmonary disease function

impairment

respiratory muscles

structure

Received and accepted June 302003
Dyspnoea, decrease of exercise capacity and impairment of quality of life are common in patients with chronic obstructive pulmonary disease (COPD). Recently, it has been demonstrated that the decrease in exercise capacity is associated with mortality of these patients. Depending on the individual, the imbalance between the functional capacity of the respiratory muscles and the load they are chronically facing plays an important role in the genesis of dyspnoea [1] and hypercapnia [2, 3]. It has also been described that respiratory muscle dysfunction could be an important determinant of increased use of health resources and survival in hospitalised patients with severe COPD [4-9].

Respiratory muscles are skeletal muscles charged with the task of expanding and compressing the chest wall. At rest or during low intensity exercise, the work of breathing in healthy individuals is relatively small and respiratory muscles have no difficulty in maintaining this level of power output. However, prolonged high-intensity exercise presents a significant challenge to respiratory muscle endurance, which can result in dyspnoea and contribute to impaired exercise tolerance. Furthermore, patients with COPD often exhibit respiratory muscle weakness and reduced respiratory muscle endurance. Respiratory muscles are submitted to multiple factors related to both the presence and severity of COPD which may impair their structure and function [10]. However, due to the fact that the diaphragm, accessory inspiratory muscles and expiratory muscles are submitted to chronic mechanical loading in COPD patients, deconditioning does not explain respiratory muscle dysfunction.

For these reasons, the interest in defining the underlying causes of respiratory and peripheral muscle dysfunction in patients with COPD has increased in the last years. According with the aims of this review, multiple factors involved in structure and function relationships of the respiratory muscle can be stratified into two groups, extrinsic and intrinsic muscle factors [11, 12]. Extrinsic factors refers to changes in chest wall geometry, pulmonary volume and systemic metabolic factors. Intrinsic factors relates to changes in fibre size, sarcomere length, muscle mass and muscle metabolism. Some previous reviews summarising the knowledge on skeletal muscles in patients with COPD are available [11-13]. The present review is aimed at highlighting some relevant recent findings and controversies regarding structure and function relationships of respiratory muscles in patients with COPD. 


\section{Factors capable of impairing respiratory muscle function and structure in COPD patients}

\section{Changes in chest wall geometry and diaphragm position}

One of the most critical factors able to impair respiratory muscle function is pulmonary hyperinflation. Shape and geometry of the chest wall is altered in patients with hyperinflation leading to a chronic reduction of the apposition zone of the diaphragm [14]. This positional change impedes three critical components of the diaphragm inspiratory action: 1) the piston-like axial displacement of the diaphragm dome, 2) the appositional action of expanding the lower rib cage, and 3 ) the insertional action of expanding the lower rib cage. Moreover, hyperinflation could change the mechanical arrangement of the crural and costal parts of the diaphragm from a series to a parallel arrangement, leading to further reduction of force-generating capacity [15]. Pulmonary hyperinflation decreases the length of the diaphragm as is demonstrated in chest radiographs [16] or spiral computed tomography [14]. It is often argued that hyperinflation can induce and increase the diaphragmatic radius of curvature, which, according to Laplace's law, would reduce the conversion of tension into pressure. However, these changes appear to be of minimal relevance in humans [17]. The effect of hyperinflation on other inspiratory muscles such as intercostals, scalenes and sternomastoids is more difficult to study, but the increased diameter of the chest wall can also limit the mechanical effectiveness of the rib cage inspiratory muscles as can be deduced following the Laplace's law. In addition, based on animal models the mechanical effectiveness of the parasternal intercostals (the most important inspiratory part of the intercostal musculature) can be decreased due to a change in the angle between the parasternal muscle fibres and the sternum ( $\beta$ angle) [18].

\section{Deleterious shortening of diaphragm sarcomere length}

Diaphragm fibre length is an important determinant of force generating capacity. The optimal length $\left(\mathrm{L}_{\mathrm{o}}\right)$ is determined by the intrinsic sarcomere length/tension relationship of the muscle. Pulmonary hyperinflation can shorten most of the diaphragm fibre sarcomere displacing it from its optimal $\mathrm{L}_{\mathrm{o}}$ precipitating a decrease in the mechanical efficiency of the muscle, as a whole [19]. This factor appears to be relevant mainly during acute dynamic hyperinflation (e.g. during exercise) once expiratory flow limitation is present. This interposition of acute-on-chronic hyperinflation can further worsen the force capacity and endurance of the diaphragm.

\section{Local activation of proteases in the respiratory muscles}

Inspiratory loading associated with diaphragm injury in humans [20], and COPD patients show increased susceptibility to additional muscle injury [20]. Experimental evidence has suggested that activation of muscle proteases is implicated in this structural injury. Calpain, a thiol protease found in the cytosol, can degrade structural proteins such as desmin and actin in skeletal muscle. Increased activity of this enzyme and alteration of potential substrates may be responsible for the early stages of degradation of muscle after increased contractile activity. In animal models, the activity of calpain appears to increase early as shown in the diaphragm from loaded animals over control levels after 1 day of tracheal banding [21]. Both high and moderate mechanical loading are able to induce a significant increase of calpain-like activity loading in the respiratory muscles of animals [22]. The diaphragm of respiratory loaded hamsters is more susceptible to calpain degradation, showing muscle injury and inflammation [23]. It is apparent that the primary mechanism underlying the increase in calpain-like activity induced by respiratory loading could be the redistribution of a soluble (free) pool of the enzyme or activation of a previously bound inactive pool [21]. These early degradative alterations contribute to ultrastructural changes such as disruption of myofibrillar apparatus, including Z-band streaming, and increased permeability of the sarcolemma [24]. The time course of diaphragm injury induced by tracheal banding and observed at the lightmicroscopic level may be similar to that observed in limb muscles.

Examination of the mediators and time course of diaphragm injury during acute (e.g. exercise) and chronic (e.g. COPD) loading would identify the critical time points of maximal diaphragm muscle deterioration and provide some guidance about the optimal time point to investigate the impact of interventions aimed at preventing respiratory muscle injury and ventilatory failure.

\section{Deleterious oxidative stress on the respiratory muscles}

In both healthy and diseased humans, skeletal muscle activation results in an increased production of free radicals and other forms of reactive oxygen species (ROS). The term oxidative stress refers to the structural damage produced by the oxidation of different components (proteins, lipids, nucleic acids) due to the presence of pro-oxidant substances [25]. Mitochondria, and both the xanthine oxidase and prostanoid pathways are the potential sources for ROS production during contractile activity [26]. Production of ROS during exercise can contribute to redox disturbances in the respiratory muscles leading to fatigue, oxidative injury, and altered expression of redox-sensitive genes. Because exerciseinduced oxidative stress can induce substantial muscle alterations $[27,28]$, it may be one of the mechanisms involved in respiratory muscle injury in patients with COPD. Therefore, given that exercise can greatly increase ROS production and upset muscle redox balance, skeletal muscle contains "redoxbuffers" to minimise exercise-induced muscle damage. The principal strategies to prevent ROS-induced injury include conversion of ROS into less active molecules (i.e. scavenging) and prevention of the transformation of less active ROS into more damaging forms (e.g. hydrogen peroxide into hydroxyl radicals). Specifically, two classes of endogenous anioxidants (enzymatic and nonenzymatic) work in tandem to decrease the harmful effects of ROS in cells. Primary antioxidant enzymes in skeletal muscles include superoxide dismutase, glutathione peroxidase, and catalase [29].

Exercise-induced oxidative stress results from an imbalance between free radical generation and the efficiency of antioxidant agents. The few studies evaluating local muscle oxidative stress have shown that strenuous incremental cycle exercise, and even light constant cycle exercise, results in oxidative stress in COPD patients [30, 31]. Although these studies focused on whole body exercise and did not identify the specific source of this exercise-induced oxidative stress (i.e. lungs, heart, liver, etc.), this finding suggests that COPD patients may be frequently exposed to lipid peroxidation and muscle damage in their daily living activities. CouILlard et al. [32] have demonstrated exercise-induced systemic oxidative stress in patients with COPD during the specific quadriceps endurance test. The observation of a significant increase in plasma products of lipid peroxidation in COPD patients $6 \mathrm{~h}$ after local quadriceps exercise indicated exerciseinduced oxidative stress in the patients. Although the clinical 
relevance of this finding needs to be elucidated, it indicates that patients with COPD present a greater susceptibility to local exercise-induced oxidative stress. If this oxidative stress originates within the muscle, it may be of clinical relevance since muscle oxidative stress generated during exercise might be one of the mechanisms of muscle dysfunction described in patients with COPD.

Two mechanisms linking exercise and oxidative stress are increased pro-oxidant activity and inadequate antioxidant activity. The activity of the superoxide anion $\left(\mathrm{O}_{2} \cdot{ }^{-}\right)$release in circulating phagocytes has been found to remain unchanged after local exercise in COPD patients. This result is not surprising since the circulating catecholamines generated during local exercise are not sufficient to induce an increase in phagocyte activation [33]. In this sense, the local exerciseinduced oxidative stress in COPD patients may not be attributable to increased phagocyte $\mathrm{O}_{2} \cdot{ }^{-}$release in response to exercise. Other mechanisms may include an altered mitochondrial respiratory chain or xanthine oxidase activity. HEUNKS et al. [31] showed that both exercise-induced glutathione oxidation and elevation of lipid peroxides were prevented by allopurinol treatment, a xanthine oxidase upregulator. This strongly suggests that xanthine oxidase, mainly localised in capillary endothelium, is involved in the exercise-induced oxidative stress in COPD patients. Apart from increased muscular oxidants, a decrease in antioxidative defences may contribute to oxidative stress induced by exercise. A deficiency in total plasma antioxidant activity has been described in COPD patients at rest [34]. Accordingly, a significant decrease in vitamin $\mathrm{E}$ levels has been found in patients with COPD [32]. ENGELEN et al. [35] showed a significant decrease in quadriceps glutathione (GSH) level in patients with emphysema, and RABINOVICH et al. [36] reported that patients with COPD had a reduced ability to adapt to endurance training, as reflected by a lower capacity to synthesise GSH.

These recent studies suggest that at least another antioxidant system may be exceeded during exercise and involved in local exercise-induced oxidative stress in these patients. It should be emphasised, however, that it has not been established whether respiratory muscles disclose a preserved or impaired redox status.

\section{Deleterious effects of malnutrition on the respiratory muscles}

Regarding the respiratory muscles, malnutrition has been found to decrease respiratory muscle strength and endurance [37-40]. Although some COPD patients showed a clearly reduced muscle mass, many of them only reveal subclinical nutritional abnormalities. Dysfunction of the respiratory muscles can also be related to the impairment in the cellular membrane transport in muscles and would preceed mass loss. Malnutrition itself can explain some of the muscle changes observed in COPD because it relates to enlargement of the relaxation ratio, loss in muscle mass, reduction in fibre size, decrease in the percentage of type II fibres and depletion of energy-rich compounds. The frequent observation that a sufficient level of food intake can coexist with progressive weight loss in some COPD patients supports the hypothesis of a relevant role for nutrient consumption related to increased respiratory muscle work and/or systemic inflammation. Malnutrition mainly affects the activity of glycolytic enzymes but does not impair the function of oxidative pathways, exactly the opposite of what has been observed in the lower limb muscles of COPD patients. There exists a general feeling that although malnutrition can be implicated in respiratory muscle dysfunction, its role is only clinically relevant in some of the patients.

\section{Deleterious effects of ageing and systemic factors}

Comorbidity, semi-starvation, inflammatory mediators and ageing are external factors which could possibly confound a direct relationship between the lung impairment and alterations in respiratory and peripheral muscles in COPD patients. Reducing the catabolic effects of the various contributing factors may improve respiratory muscle function in these patients. A systematic overview of human studies investigating alterations in skeletal muscle function, morphology and metabolism in COPD has been published recently by FRANSSEN [41] in which the author also addresses data obtained from muscle alterations in other diseases (i.e. anorexia nervosa, disuse or inactivity and ageing). In addition, a summary of the available information supporting the concept of "systemic inflammation" and its potential role in skeletal muscles is available from AGUSTí et al. [42].

\section{Evidence of adaptive changes in respiratory muscles of COPD patients: a new concept with structural basis}

Many of the studies dealing with factors contributing to inspiratory muscle dysfunction in COPD have focused on the diaphragm. This can be justified by a number of reasons [12]. First, the diaphragm is the main inspiratory muscle in humans. Secondly, it is the only inspiratory muscle that can be readily studied separately in vivo. And thirdly, hyperinflation, a characteristic feature of COPD, is particularly detrimental to the diaphragm [43-46]. Although the other inspiratory muscles have been investigated less systematically, their function becomes increasingly more important to ensure adequate ventilation as the diaphragm's function deteriorates. Accordingly, information derived from other muscles such as the latissimus dorsi, deltoid and abdominal external oblique has become useful for performing comprehensive analyses on the potential physiopathological basis and remodelling capacity of the respiratory muscles in COPD patients. In this review, the most relevant findings on diaphragm, external intercostals, latissimus dorsi, external oblique and deltoid muscles are summarised.

\section{Adaptive changes in the diaphragm}

In humans, the diaphragm is chronically active and is among the most aerobically adapted skeletal muscles. Indeed, diaphragmatic aerobic capacity and capillary density exceeds the values measured in antigravity limb muscles and approach the values measured in the myocardium. Hence, even in relatively inactive humans, the diaphragm has a relatively high aerobic capacity. In COPD patients, the diaphragm muscle works against increased mechanical loads due to airflow limitation and geometrical changes of the thorax derived from pulmonary hyperinflation. It has been hypothesised that such a loading can emulate chronic endurance training. Adaptive changes cannot be predicted from the knowledge from peripheral muscles or healthy subjects. In fact, mechanical loading in COPD is concomitant with other numerous stimuli related to the environment (electrolyte status, pro-inflammatory mediators, growth factors, etc.).

Interestingly, SIMILOWSKY et al. [43] demonstrated that COPD patients with chronic hyperinflation developed higher maximal transdiaphragmatic pressures than normal subjects 
when twitch stimulations were applied at equivalent lung volumes. In other words, the diaphragm of these patients preserved or even increased the intrinsic properties to generate pressure even though the function of the muscle was impaired because of extrinsic factors (i.e. lung volume and geometry and configuration of the chest wall). Such a finding implies that some structural adaptation occurs in the muscle. However, there is a paucity of data that allows us to establish a cause/effect relationship between presence or severity of COPD and these structural changes within the diaphragm in humans. In fact, there are obvious ethical and practical difficulties for in vivo accessing of the diaphragm of healthy subjects or stable COPD patients, unless patients are undergoing thoracotomy for other reasons. Despite these methodological limitations, a plethora of data demonstrate that the diaphragm shows remodelling changes in the face of COPD [44]. The current authors' group [45] demonstrated that length of the sarcomere from the costal diaphragm is adapted in association with the degree of pulmonary hyperinflation. Specifically, the higher the degree of intrathoracic gas volume and residual volume (RV)/total lung capacity (TLC) ratio, the shorter the length of sarcomeres. Using similar cross-sectional approaches, several authors [46-48] have been able to show an association between severe COPD and additional structural changes in the diaphragm: 1) a fast-to-slow transformation in myosin heavy chain isoforms and important myofibrillary regulatory proteins (myosin light chains, troponin subunits, and tropomyosins), 2) an increase in the proportion of type I fibres, 3) decreases in the cross-sectional area of slow (type I) fibres, and a tendency of type II fibres to show a decrease in cross-sectional area, 4) marked increases in the diaphragmatic area fraction of type I fibres and an accompanying decrease in the area fraction of type II fibres, 5) increases in mitochondrial volume density, and 6) increases in the mitochondrial oxidative capacity of all fibre types. These findings are morphological indicators of aerobic adaptation of the diaphragm in the face of the disease [44-46]. The current authors agree with other authors who suggest that, conceptually, the diaphragmatic myofibres are continuously working against the increased mechanical overloading of COPD [44, 46, 47]. This increased activity has gone on for many years, and therefore, the structural remodelling changes represent chronic adaptations [44].

\section{Adaptive changes in the external intercostal muscles}

External intercostals show electrophysiological activation during both quiet breathing and respiratory loading [49-51]. Accordingly one would presumably expect the external intercostal muscles to already be trained to a large extent in COPD patients with a baseline increase in type I fibres, as has been shown in the diaphragm. However, several previous studies have demonstrated that this is not the case for the external intercostals. External intercostals in COPD patients do not express a systematic aerobic phenotype when compared to healthy subjects. SAULEDA et al. [52] found no differences in the proportion of the fibre types in the external intercostal muscles of patients with and without airflow obstruction. This absence of switching through a type I fibre predominancy was confirmed in a subsequent study [53]. Proportions of type I and type II fibres from the external intercostal muscles of severe COPD patients (forced expiratory volume in one second (FEV1) $13-38 \%$ pred) oscillated between $51 \pm 4$ and $49 \pm 5 \%$, respectively, at the dominant side. No differences were found when compared with the external intercostals from the nondominant side, which showed $52 \pm 4$ and $48 \pm 4 \%$ type I and type II fibres, respectively. Although
SANCHEZ et al. [54] showed that the external intercostals express a $64 \pm 10 \%$ of type I fibres, this proportion was not related with either pulmonary function or nutritional status of the patients. In a complimentary study using sodium dodecylsulphate-polyacrylamide gel electrophoresis (SDS-PAGE) of proteins obtained from external intercostal extracts, a significant increase in the proportion of type II myosin heavy chain $(\mathrm{MyHC})$ and lower proportion of type I MyHC has been found in the external intercostals of severe COPD patients [55]. These findings are in some manner related to a previously published study showing that oxidative activity is preserved in the external intercostals of COPD patients, whereas activity in the glycolytic pathway seems to increase and this increase is proportional to the severity of COPD [56]. At present, it is not possible to precisely define why the external intercostal muscles express a different pattern of "change" in COPD patients when compared with the diaphragm. Since the phenotype of the intercostal muscles is not the same as that observed in the diaphragm, it has been proposed that such a particular phenotype is probably the expression of a combination of adaptive structural factors in the face of both constant (airflow limitation) and intermittent loadings (e.g. exercise, dynamic hyperinflation). These findings call for compartmentalised changes in the respiratory muscles from patients with COPD $[57,58]$.

\section{Adaptive changes in the latissimus dorsi muscle}

Most studies evaluating the structure of the respiratory muscles include the latissimus dorsi as a control muscle by assuming that it does not participate in respiratory movements. Located in the posterior region of the thorax, this muscle participates fundamentally in the adduction of the arm. In contrast, electrophysiological [59] and structural [60] studies demonstrate that this muscle participates in the ventilatory efforts in healthy and COPD patients. Activation of the muscle linearly increases in the face of inspiratory loads. This activation would reflect a potential intermittent recruitment able to induce structural changes in the latissimus dorsi of COPD patients. In this line, fibres of the latissimus dorsi show an increased diameter in COPD patients keeping a direct relationship with the severity of airflow obstruction. However, functional studies specifying the physiological translation of these structural changes are lacking.

\section{Adaptive changes in the deltoid muscles}

In a recent study assessing the metabolic pathways of the deltoid (a muscle of the upper limbs), the oxidative capacity of muscle homogenates was found to be preserved or even increased in severe COPD patients [61]. It is not clear why these findings are different to those previously observed in muscles from the lower limbs, a crucial point when evaluating the causes thought to be involved in modulating the changes of the peripheral muscles in relation to the disease. In a subsequent study, HERNÁNDEZ et al. [62] showed that although the mean size of the deltoid fibres is found to be similar between COPD and controls, important differences become evident when a more sophisticated mathematical analysis is performed. Typically, opposite histomorphometrical changes are simultaneously found in the deltoid from COPD patients, as expressed by the coexistence of atrophic and hypertrophic fibre populations. Biopsies from nondominant deltoid muscle were obtained and processed for morphometric analysis of the fibre types. Both type I and type II muscle fibres were distributed in the typical mosaic pattern. The mean value of 
the fibre size was within the normal range. However, three differentiated modes were observed in the deltoid from COPD patients: a central mode of normal sized fibres, a mode of atrophic fibres and a mode of hypertrophic fibres. This observation was evident even within single fascicles and especially prevalent in the most severe COPD patients. These findings support the theory that factors with opposite effect (promotion of either atrophy or hypertrophy) exert relevant roles in the histomorphometrical characteristics of the deltoid muscles in COPD patients [62].

\section{Expiratory muscle function and structure in chronic obstructive pulmonary disease: a missing link?}

Four abdominal muscles (external oblique, internal oblique, anterior and transverse) are considered the main expiratory muscles (ExM). Their contraction compresses the abdominal content and makes the costal ribs descend. Therefore, intrathoracic pressure increases and the diaphragm is pushed towards the thorax allowing expiratory flow to settle and the lung volume to diminish [63]. Contraction of the ExM makes the execution of expulsive efforts (such as cough, a fundamental mechanism for clearing the airways) possible. In healthy individuals, activity of these muscles also increases during exercise in proportion to ventilatory demands [64]. The ExM may play an important role in the presence of pulmonary diseases [65]. Recruitment of ExM has been observed in patients with airflow limitation, such as COPD or asthma [65-67] although the clinical significance of this activation is unclear. NiNANE et al. [65] demonstrated that COPD patients recruit the ExM, even during breathing at rest, according to the severity of airflow obstruction. Although the clinical significance of this activation is unknown, contraction of the ExM might represent a compensatory mechanism in obstructive lung diseases [67]. The ExM contraction can store both elastic and gravitational energy within the thorax and abdomen, facilitating the beginning of the following inspiratory cycle [67]. In this line, it seems reasonable to hypothesise that airflow obstruction can induce adaptive changes in the ExM of COPD patients. However, RAMIREZ-SARMIENTO et al. [68] showed that both strength and endurance of ExM is significantly decreased in COPD patients. Weakness and susceptibility to ExM fatigue cannot be explained by geometrical changes of the thorax (i.e. pulmonary hyperinflation). All this information allows us to suggest that deconditioning of the expiratory muscles appears improbable. Some preliminary studies on the structure of the ExM provide evidence that fibre size and type distribution within the muscles can be modified in association with presence and severity of the disease. A promising line of investigation is the evaluation of specific expiratory muscle training as a potential therapeutic measure to improve expiratory muscle function in COPD.

\section{Clinical implications of impairment versus adaptation of the respiratory muscles in COPD: the "fragile balance"}

At present, literature supports that factors able to impair the function and the structure of the respiratory muscles coexist with a capacity of the muscles to show adaptive changes. From a clinical point of view, however, this adaptation is insufficient to restore normal strength and endurance of the muscles. In fact, this particular balance between adaptation and many opposite factors results in a decreased mechanical capacity of the respiratory muscles, loss of muscle mass, and an increased susceptibility to respiratory muscle fatigue and injury.
Chronic obstructive pulmonary disease and decreased mechanical capacity of respiratory muscles

Strength of the inspiratory muscles has been consistently demonstrated to be decreased in patients with COPD when compared with healthy individuals [47]. This alteration has been associated with mechanical disadvantage and changes in muscle length due to pulmonary hyperinflation. This mechanical disadvantage has conventionally been defined as weakness of inspiratory muscles. Weakness can be evidenced when the assessment is performed through voluntary manoeuvres (e.g. maximal inspiratory pressures $(P \mathrm{I}, \max ))$. Measurements can be performed at the mouth (PIm,max) or oesophagus $(P$ es,max $)$. These findings indicate that the capacity of the inspiratory muscles to act as a group to generate negative intrathoracic pressure at RV or functional residual capacity (FRC) is reduced in the patients. Similarly, strength of the diaphragm as assessed by the transdiaphragmatic pressure $(P$ di) during sniff or Müller manoeuvres ( $P$ dimax,sniff or $P$ dimax,Müller, respectively) is usually decreased in COPD patients. This functional alteration would not be justified for a decreased central drive. The $P$ di elicited at FRC by phrenic stimulation, either electric or magnetic, is also reduced in these patients [69]. The current authors feel that it is important to highlight that most studies have interpreted muscle strength as assessed by pressure measurements. At least for the diaphragm, the assumption that inspiratory pressure is a reflection of inspiratory muscle strength can be misleading because the relationship between tension developed by the muscle fibres versus pressure measured at the mouth or oesophagus is not necessarily constant. Regarding the expiratory muscle function, expiratory muscle strength (as assessed by maximal static expiratory pressure has been found to be relatively preserved [70] or decreased [16, 71]. In addition, RAMIREZ-SARMIENTO et al. [68] demonstrated that endurance of the expiratory muscles is also decreased in patients with COPD. Deterioration in ExM endurance was found to be associated with the severity of the disease and is associated with lower strength in different muscle groups. This suggests that systemic factors can be implicated in the origin of these impairments.

\section{Chronic obstructive pulmonary disease and changes in respiratory muscle mass}

Despite the above-mentioned mechanistic explanation of diaphragm dysfunction in COPD, there is growing evidence for intrinsic adaptation within the muscle. To determine whether the lower $P \mathrm{I}$,max in a COPD group (including both normal weight and underweight patients) is due to hyperinflation, SAHEBJAmi et al. [72] corrected $P$ I,max values for the RV/TLC ratio. They showed that the lower $P \mathrm{I}$,max in the underweight subjects with COPD is most likely due to muscle weakness rather than mechanical factors [72]. Several studies have shown that size (diameter or area) of the fibres is decreased in the diaphragm of patients with COPD [45, 46]. In emphysematous patients, a positive correlation was found between diaphragm mass and the pathologic severity score of emphysema [73]. This finding is consistent with the concept of muscle fibre atrophy. Due to the organisation of actin-myosin components in a constant spatial fashion, fibre atrophy would represent a decrease in the content of contractile proteins in the diaphragm from COPD. A decrease of myosin and actin cross-bridges conditions a decrease in muscle tension generation and strength. However, a decreased muscle fibre size could also represent an adaptive phenomenon to facilitate diffusive and convective oxygen transport from the capillary 
network towards the cytosolic and mitochondrial enzymatic machinery [45, 46]. Furthermore, it can be questioned if diaphragm atrophy is a typical feature of COPD. A recent analysis was performed on diaphragm biopsies obtained from $>50$ patients including a complete spectrum of COPD severity showing no comorbidity. Size of the diaphragm fibres (diameter and cross sectional area) was found to be preserved independently of COPD when compared with controls. This absence of atrophy was found even in patients with the most severe airflow obstruction and pulmonary hyperinflation. This finding leads to the idea that atrophy of the diaphragm should be considered an effect of comorbidity (e.g. chronic starvation), or treatments (e.g. steroids) more than a direct consequence of the mechanical loading due to the COPD per se.

\section{Chronic obstructive pulmonary disease and susceptibility to respiratory muscle fatigue}

Although this concept is not covered in depth in the literature, several studies show that endurance of the inspiratory muscles is decreased in patients with COPD. Endurance would probably be a determinant in tolerating workload and increasing ventilation while exercising or during exacerbations of COPD. In the context of neurophysiology, fatigue represents a decrease in muscle force in response to a given neural stimulus. Contractile fatigue develops when the respiratory system is challenged with an excessive mechanical load for an extended duration. Contractile fatigue can be shortlasting or long-lasting. Short-lasting fatigue, also known as high-frequency fatigue, results from accumulation of inorganic phosphate, failure of the membrane electrical potential to propagate beyond T-tubes, and to a lesser extent intramuscular acidosis. Long-lasting fatigue, also known as lowfrequency fatigue, is consistent with the development of, and recovery from, muscle injury, and it can persist for days. The present authors' agree with most authors that endurance of the respiratory muscles is probably more relevant than maximal force [74]. In fact, breathing is a lifelong act in both healthy and diseased beings. However, guidelines for endurance tests have not gained widespread inclusion in clinical settings [74]. Endurance tests are more difficult to perform and interpret than strength measurements. These tests imply normalised patient stimulation and a high degree of patient motivation. Exhaustion during the tests could be difficult to define and to detect by nonexperienced evaluators. However, the outcome variables obtained from endurance tests are extremely valuable because they integrate psychological and perceptual factors (dyspnoea, effort) as well as metabolic and structural characteristics from exerted muscles. Several authors have demonstrated that endurance tests can be reliable and potentially valid to use in clinical assessment. Accordingly, it has recently been demonstrated in a clinical trial that specific respiratory muscle training is able to improve respiratory muscle endurance. Interestingly, the improvement was concomitant with structural changes in the trained muscles (see below). Recent guidelines for evaluating respiratory muscle endurance are available.

\section{Chronic obstructive pulmonary disease and susceptibility to respiratory muscle injury}

Chronic hypercapnia in ventilatory failure may be at least partially attributable to respiratory muscle dysfunction caused by fatigue [75], weakness [76] or injury [77] of the respiratory muscles. REID et al. [78] found that significant diaphragm injury and hypercapnic ventilatory failure are induced by resistive loading over a 6-day period in the hamster. Cell necrosis, cytoplasmic fragmentation, and an increased nuclearity can be found within and between degenerating fibres as signs of muscle injury. The current authors' group has recently demonstrated that the human diaphragm is susceptible to suffering injury in the face of inspiratory loading [20]. In this study, subjects with normal lung function and patients with COPD were submitted to one session of a specific inspiratory muscle endurance prior to thoracotomy for lung neoplasm. This study provides the first evidence that diaphragm muscle injury can be precipitated in humans during or after acute, high intensity inspiratory loading. Of interest, the diaphragm of COPD patients not only showed greater injury but also greater susceptibility to additional injury during and/or after inspiratory loading. The elucidation of potential factors leading to diaphragm injury in humans may help determine the aetiology of respiratory muscle dysfunction observed during clinical stability and acute respiratory failure. The potential effects of such injury on muscle function, remodelling mechanisms, and clinical outcomes warrants further studies. Defining the relationships between muscle dysfunction, muscle injury, and adaptive processes will facilitate the development of more effective training regimens in the context of pulmonary rehabilitation and may be important in the critical care setting when weaning patients from mechanical ventilation.

\section{Response of respiratory muscles to additional loading: injury versus training effect}

Weakness and deconditioning of respiratory and peripheral muscles are currently recognised as factors implicated in the reduction of exercise capacity as well as quality of life of COPD patients [79-82]. It is probable that inspiratory muscle dysfunction does not limit minimal ventilatory needs at rest but it does appear to contribute to dyspnoea, decreased exercise capacity and ventilatory failure during exacerbations [83]. For these reasons, specific respiratory muscle training could be justified as a strategy with potential clinical benefits in stable COPD patients who remain symptomatic despite optimal therapy [84]. On the other hand, evidence that even a single bout of inspiratory loading can induce respiratory muscle injury in COPD patients also exists. Exercise-induced muscle injury is associated with morphological abnormalities such as degeneration of the cytoplasm, disruption of cell membraneous structures (sarcolemma, mitochondria, sarcoplasmic reticulum and T-tubules), and disorganisation of the contractile myofibrils (including Z-band streaming, misalignment of the myofilaments, and desmin loss) [85]. How can the existence of these two phenomena be integrated? Although this opposite effect could appear controversial, a comprehensive hypothesis is that respiratory muscle training may be the result of repetitive bouts of muscle injury followed by regenerative mechanisms and adaptation. Although exertion-induced injury is associated with impaired muscle function (decreased strength and/or endurance), muscle injury also appears to stimulate complex mechanisms that can induce adaptive repair to increased utilisation and stress in skeletal muscles (i.e. training) [86, 87]. A summary of findings supporting these concepts appears below.

\section{Loading and respiratory muscle injury}

Muscle injury is a term that refers to the structural damage of different muscle components (mitochondria, sarcolemma, 
sarcomere) due to muscle activity itself or other multiple factors (oxidative stress, inflammation, malnutrition, toxics, etc.). Muscle injury occurs in a complex interplay of events. Initially, muscle tension may actively disrupt muscle fibre membranes and the cytoskeleton, including the Z-line structure. Subsequent damage can result from an increase in intracellular calcium concentration, which activates proteases like calpain. Both myofilament regulatory and structural proteins are vulnerable to cleavage and loss as a result of calpain degradation. Some aspects of the inflammatory process are triggered early in exertion, such as an increase in the number of circulating blood cells and upregulation of neutrophils. Neutrophils may be observed during the first 24-h after a bout of muscle overload. However, significant injury of the muscle observed under the light microscope may take two or three more days. The observed changes include fibre necrosis, infiltration of mononuclear cells, and regenerative changes. The tendency for diaphragm injury to be less severe at 4 days after loading may reflect an increase in regeneration [21, 77].

Recently published data show that the diaphragm from COPD patients discloses signs of injury even in spite of clinical stability. In a cross-sectional study, MACGowAN et al. [88] recently showed that the degree of airflow obstruction (i.e. per cent predicted FEV1) is inversely related to the abnormal muscle and directly related to the area fraction of normal muscle. Inflammatory cells (macrophages) were found in most of the diaphragm samples although the number of cells were not related to FEV1. This study demonstrated that increasing severity of airflow obstruction is associated with an increased proportion of abnormal (injured) diaphragm and a decreased proportion of normal diaphragm fibres. Using electron microscopy, a recent clinical trial from the current authors' group [20] showed that sarcomere disruptions (a sign of muscle injury) is present in the diaphragm of both healthy controls and COPD patients. The ranges of sarcomere disruption values were wide and higher in the diaphragm from COPD (area fractions: 1.3 to $17.3 \%$ ). In addition, sarcomere disruption was higher (in both groups) when the subjects were submitted to a single bout of inspiratory loading, with the greatest increase of injury in the diaphragm of COPD patients. From a clinical point of view, this study indicates that 1) sarcomere disruption is a common (physiological) finding in the human diaphragm, 2) sarcomere disruptions are abnormally increased in the diaphragm of COPD, and 3) susceptibility to additional respiratory muscle injury is significantly increased $(\approx 300 \%)$ in these patients [20].

\section{Loading and respiratory muscle training}

Several functional studies in both healthy individuals and COPD patients have demonstrated that repetitive inspiratory muscle loading (i.e. inspiratory training) can increase the strength and endurance of inspiratory muscles [89, 90, 91]. This functional improvement is observed only when specific inspiratory muscle training is performed, but not when general exercise programmes are applied [84]. Some authors have demonstrated that this training may have a general impact in terms of exercise capacity, endurance time on a treadmill, or dyspnoea [90, 92-95]. However, other studies have been unable to show any changes in either walking distance or maximal oxygen uptake [91, 96-98]. Other authors have reported no significant changes in inspiratory muscle function following specific training [99]. The controversy appears to be related to the differences in either the magnitude or duration of inspiratory muscle loading [100]. Inspiratory muscle training has been found to be capable of improving inspiratory muscle function when intensity is monitored and exceeds $20 \%$ of PIm,max [101, 102]. Recently, for the first time in a clinical trial, RAMIREZ-SARMIENTO et al. [103] evaluated the structural changes in the respiratory muscles of COPD patients after a specific program of respiratory muscle training. Increases were observed in both the proportion of type I fibres $(\cong 38 \%)$ and the size of type II fibres $(\cong 21 \%)$ of a group of inspiratory muscles (i.e. external intercostals) following the training period. From a clinical point of view, this study highlights three main concepts. Firstly, the inspiratory muscles of COPD patients preserve a capacity to show remodelling (conditioning) changes following a short-term loading period [103]. The muscles exhibit a classical response to training that would be predictable in limb muscles. Similar findings have been described in the peripheral muscles of COPD patients following general muscle training [104]. The second point relates to syncronic functional and structural changes. The increase in inspiratory muscle endurance and strength after specific training appears to be related to switching of $\mathrm{MyHC}$ isoforms (as assessed by the increase in fibres expressing MyHC-I) and the increase in fibre size (mainly in type II fibres) [103]. Other factors, such as perceptual adaptation to additional inspiratory loading (e.g. dyspnoea desensitisation), learning of specific manoeuvres, or even a placebo effect, could also participate in improving inspiratory muscle strength and endurance. Finally, the study highlights training specificity. The inspiratory training showed specific functional and structural effects only on the trained (inspiratory) muscles [103]. Further studies assessing the potential role of respiratory muscle training on quality of life and survival of COPD patients appears to be warranted.

\section{A comprehensive analysis: the "theory of muscle compartments"}

Taking all these findings together, it can be stated that respiratory muscles show both functional and structural changes in COPD patients. However, available data support the idea that these changes differ not only in magnitude but also in sense according to the muscle or group analysed. These changes are particular for each muscle territory or compartment (table 1). The diaphragm of patients with COPD presents several adaptative changes not recorded in peripheral muscles. Some accessory respiratory muscles show fibre hypertrophy while other muscles consistently show a decrease in the size of their fibres. On the other hand, muscles of the lower limbs disclose different changes to those observed in upper limb muscles. These findings imply that functional impairment and structural changes are not homogeneous in all the respiratory or limb skeletal muscles in patients with COPD. Moreover, the final balance between impairment or adaptive changes within a muscle group varies interindividually between the patients and cannot be predicted from conventional pulmonary function tests. From a practical point of view, these concepts emphasise that respiratory muscle abnormalities in patients with COPD should be addressed directly rather than deduced from data obtained by assessing peripheral muscle structure or function. In addition, the global concept of "peripheral muscles" is misleading, since these have to be grouped in at least two different compartments: upper and lower limb muscles. The structure and function are relatively preserved in the former possibly due to the maintenance of some daily activities involving the arms or even the use of some of these muscles in the ventilatory effort. In contrast, involutional structural changes prevail in the lower limbs which results in an impairment of the limb function and in the global exercise 
capacity of the individual. A progressive deconditioning due to a reduction in daily activity is probably the driving force for changes in the quadriceps muscle. Although the level of activity appears to be the main determining factor in changes occurring in different territories, this appears to be enhanced or counterbalanced by other local and systemic factors such as inflammation, oxidative stress, drugs and nutritional abnormalities.

From a practical and clinical point of view, these concepts have been summarised in the "theory of muscle compartments" which implies [57, 105] firstly, the requirement to include more specific tests in order to evaluate the strength and endurance in each muscle group or compartment, and secondly, the possibility to improve the rehabilitation outcomes by applying personalised conditioning strategies of the respiratory muscles. In order to implement correct comprehensive evaluation of respiratory muscle function, it is imperative that research laboratories possess the relevant equipment and methodology. It is possible that these measures would optimise the impact of the rehabilitation strategies keeping in mind the prevalence and natural history of chronic obstructive pulmonary disease, the cost of the treatment, and the restrictions of current public health policies.

\section{References}

1. Dyspnea: mechanisms, assessment, and management: a consensus statement: American Thoracic Society. Am J Respir Crit Care Med 1999; 159: 321-340.

2. Rochester DF. Respiratory muscle weakness, pattern of breathing, and $\mathrm{CO}_{2}$ retention in chronic obstructive pulmonary disease. Am Rev Respir Dis 1991; 143: 901.

3. Scano G, Spinelli A, Duranti R, et al. Carbon dioxide response in COPD patients with and without chronic hypercapnia. Eur Respir J 1995; 8: 78.

4. Gray-Donald K, Gibbons L, Shapiro SH, et al. Nutritional status and mortality in chronic obstructive pulmonary disease. Am J Respir Crit Care Med 1996; 153: 961.

5. Strauss MJ, Conrad D, Logerfo JP, Hudson LD, Berrgner M. Cost and outcome of care for patients with chronic obstructive lung disease. Analysis by physician speciality. Med Care 1986; 24: 915-924.

6. Decramer M, Gosselink R, Troosters T, Vercshueren M, Evers G. Muscle weakness is related to utilization of health care resources in COPD patients. Eur Respir J 1997; 10: $417-423$.

7. Intermittent Positive Pressure Breathing Trial Group. Intermittent positive pressure breathing therapy of chronic obstructive pulmonary disease. Ann Intern Med 1983; 99: 612-620.

8. Decramer M, Gosselink R, Troosters T, Schepers R. Peripheral muscle weakness is associated with reduced survival in COPD. Am J Respir Crit Care Med 1998; 157: A19.

9. Decramer M, De Bock V, Dom R. Functional and histologic picture of steroid-induced myopathy in chronic obstructive pulmonary disease. Am J Respir Crit Care Med. 1996; 153: 1958-1964.

10. Killian KJ, Leblanc P, Martin DH, Summers E, Jones NL, Campbell EJM. Exercise capacity and ventilatory, circulatory, and symptom limitation in patients with chronic airflow limitation. Am Rev Respir Dis 1992; 146: 935-940.

11. American Thoracic Society/European Respiratory Society. Skeletal muscle dysfunction in chronic obstructive pulmonary disease. Am J Respir Crit Care Med 1999; 159: S1-S40.

12. Marchand E, Decramer M. Respiratory muscle dysfunction and drive in chronic obstructive pulmonary disease. Clin Chest Med 2000; 21: 679-692.

13. Fitting JW. Respiratory muscles in chronic obstructive pulmonary disease. Swiss Med Wkly 2001; 131: 483-486. 
14. Cassart M, Pettiaux N, Gavenois PA, Pavia M, Estenne M. Effect of chronic hyperinflation on diaphragm length and surface area. Am J Respir Crit Care Med 1997; 156: 504.

15. Macklem PT, Mackelm DM, De Troyer A. A model of inspiratory muscle mechanics. J Appl Physiol 1997; 155: 1590.

16. Rochester DF, Braun NMT, Arora NS. Respiratory muscle strength in chronic obstructive pulmonary disease. Am Rev Respir Dis 1979; 119: 151.

17. Gauthier AP, Verbank S, Estenne M, Segebarth C, Macklem PT, Pavia M. Three dimensional reconstruction of the in vivo human diaphragm shape at different lung volumes. $J$ Appl Physiol 1994; 76: 495.

18. Ninane V, Gorini M. Adverse effects of hyperinflation on paraesternal intercostals. J Appl Physiol 1994; 77: 2201.

19. Smith J, Bellemare F. The effect of lung volume of in vivo contraction characteristics of human diaphragm. $J \mathrm{Appl}$ Physiol 1987; 62: 1893.

20. Orozco-Levi M, Lloreta J, Minguella J, Serrano S, Broquetas JM, Gea J. Injury of the human diaphragm associated with exertion and chronic obstructive pulmonary disease. Am J Respir Crit Care Med 2001; 164: 1734-1739.

21. Reid WD, Belcastro AN. Time course of diaphrgam injury and calpain activity during resistive loading. Am J Respir Crit Care Med 2000; 162: 1801-1806.

22. Jiang T-X, Reid WD, Belcastro AN, Road JD. Load dependence of secondary diaphragm inflammation and injury after acute respiratory loading. Am $J$ Respir Crit Care Med 1998; 157: 1381-1386.

23. Reid WD, Huang J, Bryon S, Walker DC, Belcastro AN. Diaphragm injury and myofibrillar structure induced by resistive loading. J Appl Physiol 1994; 76: 176-184.

24. Belcastro AN, Shewchuk LD, Raj DA. Exercise-induced muscle injury: a calcium-calpain hypothesis. Mol Cell Biochem 1998; 179: 135-145.

25. Repine JE, Bast A, Lankhorst I, and the Oxidative Stress Study Group. Oxidative stress in chronic obstructive pulmonary disease. Am J Respir Crit Care Med 1991; 144: 570-574.

26. Powers S, Criswell D, Lawler J, et al. Adaptive strategies of respiratory muscles in response to endurance training. Med Sci Sports Exerc 1996; 28: 1115-1122.

27. Barcklay JK, Hansel M. Free radicals may contribute to oxidative skeletal muscle fatigue. Can J Physiol Pharmacol 1991; 69: 279-284.

28. Davies K, Quintanihla A, Brooks G, Packer L. Free radicals and tissue damage produced by exercise. Biochem and Biophys Res Comm 1982; 107: 1198-1205.

29. Halliwell B, Gutteridge J. Free Radicals in Biology and Medicine. New York, Oxford Science Publications, 1999.

30. Vina J, Servera E, Asensi M, et al. Exercise causes blood glutathione oxidation in chronic obstructive pulmonary disease: prevention by $\mathrm{O}_{2}$ therapy. J Appl Physiol 1996; 81 : 2199-2202.

31. Heunks L, Vina $\mathbf{J}$, Cees L, et al. Xanthine oxidase is involved in exercise-induced oxidative stress in chronic obstructive pulmonary disease. Am J Physiol 1999; 277: R1697-R1704.

32. Couillard A, Koechlin C, Cristol JP, Varray A, Prefaut C. Evidence of local exercise-induced systemic oxidative stress in chronic obstructive pulmonary disease. Eur Respir J 2002; 20: $1123-1129$.

33. Katzuhiko S, Sato H, Takashi Kikuchi T, et al. Capacity of circulating neutrophils to produce reactive oxygen species after exhaustive exercise. J Appl Physiol 1996; 81: 1213-1222.

34. Rahman I, Morrison D, Donaldson K, MacNee W. Systemic oxidative stress in asthma, COPD, and smokers. Am J Respir Crit Care Med 1996; 154: 1055-1060.

35. Engelen MPKJ, Schols AMWJ, Does JD, Deutz NEP, Wouters EFM. Altered glutamate metabolism is associated with reduced muscle glutathione levels in patients with emphysema. Am J Respir Crit Care Med 2000; 161: 98-103.

36. Rabinovich RA, Ardite E, Trooster T, et al. Reduced muscle redox capacity after endurance training in patients with chronic obstructive pulmonary disease. Am J Respir Crit Care Med 2001; 164: 1114-1118.

37. Sahebjami H, Sathianpitayakul E. Influence of body weight on the severity of dyspnea in chronic obstructive pulmonary disease. Am J Respir Crit Care Med 2000; 161: 886-890.

38. Rochester DF, Arora NS, Braun NMT. Respiratory muscle strength and maximal voluntary ventilation in undernourished patients. Am Rev Respir Dis 1982; 126: 5-8.

39. Arora NS, Rochester DF. Effects of body weight and muscularity on human diaphragm muscle mass. $J$ Appl Physiol 1982; 52: 64-70.

40. Rochester DF, Arora NS, Braun NMT. Maximum contractile force of human diaphragm muscle. Trans Am Clin Climatol 1981; 93: 200-208.

41. Franssen F. The influence of comorbid factors on muscle function, morphology and metabolism in COPD. Eur Respir Mon 2003; 24: 99-112.

42. Agustí AGN, Noguera A, Sauleda J, Busquets X. Systemic inflammation in chronic respiratory disease. Eur Respir Mon 2003; 8: 24, 46-55.

43. Similowski T, Yan S, Gauthier AP, Macklem PT, Bellemare F. Contractile properties of the human diaphragm during chronic hyperinflation. $N$ Engl J Med 1991 26: 325: 917-923.

44. Levine S, Nguyen T, Kaiser RK, Shrager JB. Evaluating respiratory muscle adaptations: A new approach. Am J Respir Crit Care Med 2002; 166: 1418-1419.

45. Orozco-Levi M, Gea J, Lloreta JL, et al. Subcellular adaptation of the human diaphragm in chronic obstructive pulmonary disease. Eur Respir J 1999; 13: 371-378.

46. Levine S, Kaiser L, Leferovich J, Tikunov B. Cellular adaptations in the diaphragm in chronic obsructive pulmonary disease. $N$ Engl J Med 1997; 337: 1799-1806.

47. Levine S, Gregory C, Nguyen $\mathrm{T}$, et al. Bioenergetic adaptation of individual human diaphragmatic myofibres to severe COPD. J Appl Physiol 2002; 92: 1205-1213.

48. Mercadier JJ, Schwartz K, Sciaffino S, et al. Myosin heavy chain gene expression changes in the diaphragm of patients with chronic hyperinflation. Am J Physiol 1998; 274: L527-L534.

49. Wilson TA, Legrand A, Gevemois PA, De Troyer A. Respiratory effects of the external and internal intercostal muscles in humans. $J$ Physiol 2001; 530: 319-330.

50. Gandevia SC, Leeper JB, McKenzie DK, De Troyer A. Discharge frequencies of parasternal intercostal and scalene motor units during breathing in normal and COPD subjects. Am J Respir Crit Care Med 1996; 153: 622-628.

51. Brancatisano A, Engel LA, Loring SH. Lung volume and effectiveness of inspiratory muscles. J Appl Physiol 1993; 74: 688-694.

52. Sauleda J, Gea J, Orozco-Levi M, et al. Structure and function relationships of the respiratory muscles. Eur Respir $J$ 1998; 11: 906-911.

53. Jiménez-Fuentes MA, Gea J, Pallás O, Gallego F, Félez MA, Broquetas JM. Fibre morphometry of the external intercostal muscle. Comparison of dominant and non-dominant sides of patients with severe COPD. Arch Bronconeumol 1998; 34: 189-193.

54. Sanchez J, Brunet A, Medrano G, Debesse B, Derenne JP. Metabolic enzymatic activities in the intercostal and serratus muscles and in the latissimus dorsi of middle-aged normal men and patients with moderate obstructive pulmonary disease. Eur Respir J 1988; 1: 376-383.

55. Gea J. Myosin gene expression in the respiratory muscles. Eur Respir J 1997; 10: 2404-2410.

56. Pastó M, Gea J, Blanco ML, et al. Metabolic activity of the external intercostal muscle of patients with COPD. Arch Bronconeumol 2001; 37: 108-114.

57. Orozco-Levi M, Gea J. Muscle changes in chronic obstructive pulmonary disease: the "compartments theory". Arch Bronconeumol 2000; 36: 95-102. 
58. Gea J, Orozco-Levi M, Barreiro E, Ferrer A, Broquetas J. Structural and functional changes in the skeletal muscles of COPD patients: the "compartments" theory. Monaldi Arch Chest Dis 2001; 56: 214-224.

59. Orozco-Levi M, Gea J, Monells J, Aran X, Aguar MC, Broquetas JM. Activity of latissimus dorsi muscle during inspiratory threshold loads. Eur Respir J 1995; 8: 441-445.

60. Orozco-Levi M, Gea J, Sauleda J, et al. Structure of the latissimus dorsi muscle and respiratory function. $J$ Appl Physiol 1995; 78: 1132-1139.

61. Gea J, Pasto M, Carmona MA, Orozco-Levi M, Palomeque J, Broquetas J. Metabolic characteristics of the deltoid muscle in patients with chronic obstructive pulmonary disease. Eur Respir $J$ 2001; 17: 1-7.

62. Hernández N, Orozco-Levi M, Belalcázar V, et al. Dual morphometrical changes of the deltoid muscle in patients with COPD. Resp Physiol Neurobiol 2003; 134: 219-229.

63. Epstein SK. An overview of respiratory muscle function. Clin Chest Med 1994; 15: 619-639.

64. Fuller D, Sullivan J, Fregosi R. Expiratory muscle endurance performance after exhaustive submaximal exercise. $J$ Appl Physiol 1996; 80: 1495-1502.

65. Ninane V, Rypens F, Yernault JC, De Troyer A. Abdominal muscle use during breathing in patients with chronic airflow obstruction. Am Rev Respir Dis 1992; 146: 16-21.

66. Ninane V, Yernault JC, De Troyer A. Intrinsic PEEP in patients with chronic obstructive pulmonary disease. Role of expiratory muscles. Am Rev Respir Dis 1993; 148: 1037-1042.

67. Dodd DS, Brancatisano T, Engel LA. Chest wall mechanics during exercise in patients with severe chronic airflow obstruction. Am Rev Respir Dis 1984; 129: 33-38.

68. Ramirez-Sarmiento A, Orozco-Levi M, Barreiro E, Ferrer A, Broquetas JM, Gea J. Expiratory muscle endurance in chronic obstructive pulmonary disease. Thorax 2002; 57: 132-136.

69. Similowski T, Yan S, Gauthier AP, et al. Contractile properties of the human diaphragm during chronic hyperinflation. N Engl J Med 1991; 325: 917.

70. Morrison NJ, Richardson J, Dunn L, et al. Respiratory muscle performance in normal elderly subjects and patients with COPD. Chest 1989; 95: 90.

71. Nishimura Y, Tsutsumi M, Nakata H, et al. Relationship between respiratory muscle strength and lean body mass in men with COPD. Chest 1995; 107: 1232.

72. Sahebjami H, Sathianpitayakul E. Influence of body weight on the severity of dyspnea in chronic obstructive pulmonary disease. Am J Respir Crit Care Med 2000; 161: 886-890.

73. Thurlbek WM. Diaphragm and body weight in emphysema. Thorax 1978; 33: 483.

74. Rochester DF. Tests of respiratory muscle function. Clin Chest Med 1988; 9: 249.

75. Roussos C, Moxham J, Bellemare F. Respiratory muscle fatigue. In: C. Roussos, ed. The Thorax, Part B: Lung Biology in Health and Disease, Vol 85, Ch. 49. New York, Marcel Dekker, 1995; pp. 1405-1461.

76. Begin P, Grassino A. Inspiratory muscle dysfunction and chronic hypercapnia in chronic obstructive pulmonary disease. Am Rev Respir Dis 1991; 143: 905-912.

77. Reid WD, McGowan N. Respiratory muscle injury in humans and animal models. Mol Cell Biochem 1998; 179: 63-80.

78. Reid WD, Huang J, Bryon S, Walker DC, Belcastro AN. Diaphragm injury and myofibrillar structure induced by resistive loading. J Appl Physiol 1994; 76: 176-184.

79. Hamilton N, Killian KJ, Summers E, Jones NL. Muscle strength, symptom intensity, and exercise capacity in patients with cardiorespiratory disorders. Am J Respir Crit Care Med 1995; 152: 2021-2031.

80. Rochester DF. The respiratory muscles in COPD. State of the art. Chest 1984; 85: 47-50.

81. American Thoracic Society - European Respiratory Society. Skeletal muscle dysfunction in chronic obstructive pulmonary disease. Am J Respir Crit Care Med 1999; 159: S1-S28.
82. Arora NS, Rochester DF. Effect of chronic obstructive pulmonary disease in diaphragm muscle dimensions. Am Rev Respir Dis 1981; 123: 176-184.

83. Begin $\mathrm{P}$, Grassino A. Inspiratory muscle dysfunction and chronic hypercapnia in chronic obstructive pulmonary disease. Am Rev Respir Dis 1991; 143: 905-912.

84. ACCP/AACVPR Pulmonary Rehabilitation Guidelines Panel. Pulmonary rehabilitation: joint ACCP/AACVPR evidencebased guidelines. Chest 1997; 112: 1363-1396.

85. Reid WD, MacGowan NA. Respiratory muscle injury in animal models and humans. Mol Cell Biochem 1998; 179: 63-80.

86. Petrof BJ, Shrager JB, Stedman HH, Kelly AM, Sweeney HL. Dystrophin protects the sarcolemma from stresses developed during muscle contraction. Proc Natl Acad Sci USA 1993; 90: 3710-3714.

87. McNeil PL, Ito S. Molecular traffic through plasma membrane disruptions of cells in vivo. J Cell Sci 1990; 96: 549-556.

88. Macgowan NA, Evans KG, Road JD, Reid WD. Diaphragm injury in individuals with airflow obstruction. Am J Respir Crit Care Med 2001; 163: 1654-1659.

89. Leith DE, Bradley M. Ventilatory muscle strength and endurance training. J Appl Physiol 1976; 41: 508-516.

90. Weiner P, Magadle Rasmi, Berar-Yanay R, Berar-Yanay N, Davidovich A, Weiner M. The cumulative effect of longacting broncodilators, exercise, and inspiratory muscle training in patients with advanced COPD. Chest 2000; 118: 672-678.

91. Belman MJ, Shadmehr R. Targeted resistive ventilatory muscle training in chronic obstructive pulmonary disease. J Appl Physiol 1988; 65: 2726-2735.

92. Dekuitjzen PR, Folgering HT, Van Herwaarden CL. Targetflow inspiratory muscle training during pulmonary rehabilitation in patients with COPD. Chest 1991; 99: 128-133.

93. Larson JL, Kim MJ, Sharp JT, et al. Inspiratory muscle training with a pressure threshold breathing device in patients with COPD. Am Rev Respir Dis 1988; 138: 689-696.

94. Weiner P, Azgad Y, Ganam R. Inspiratory muscle training combined with general exercise in patients with COPD. Chest 1992; 102: 1351-1356.

95. Lisboa C, Muñoz V, Beroiza T, Leiva A, Cruz E. Inspiratory muscle training in chronic airflow limitation: comparison of two different training loads with a threshold device. Eur Respir J 1994; 7: 1266-1274.

96. Noseda A, Carpieux JP, Vandeput W, et al. Resistive inspiratory muscle training and exercise performance in COPD patients. A comparative study with conventional breathing training. Bull Eur Respir Physiopathol Respir 1987; 23: 457-463.

97. Flynn MG, Barter CE, Nosworthy JC, et al. Threshold pressure training, breathing pattern, and exercise performance in chronic airflow obstruction. Chest 1989; 95: 535-540.

98. Madsen F, Secher NH, Kay L, Kok-Jensen A, Rube N. Inspiratory resistance versus general physical training in patients with chronic obstructive pulmonary disease. Am Rev Respir Dis 1985; 67: 167-176.

99. Guyatt G, Keller J, Singer J, Halcrow S, Newhouse M. Controlled trial of respiratory muscle training in chronic airflow limitation. Thorax 1992; 47: 598-602.

100. Donner CF, Muir JF. Selection criteria and programmes for pulmonary rehabilitation in COPD patients. Rehabilitation and Chronic care scientific group of the European Respiratory Society. Eur Respir J 1997; 10: 744-757.

101. Belman MJ, Shadmehr R. Targeted resistive ventilatory muscle training in chronic pulmonary disease. J Appl Physiol 1988; 65: 2726-2735.

102. Heijdra YF, Dekhuijzen PNR, Van Herwaarden CLA, Folgering HTM. Nocturnal saturation improves by targetflow inspiratory muscle training in patients with COPD. Am J Respir Crit Care Med 1996; 153: 260-265.

103. Ramirez-Sarmiento A, Orozco-Levi M, Güell R, et al. 
Inspiratory muscle training in patients with chronic obstructive pulmonary disease. Structural and physiologic outcomes. Am J Respir Crit Care Med 2002; 1666: 1491-1497.

104. Maltais F, Leblanc P, Simard C, et al. Skeletal muscle adaptation to endurance training in patients with chronic obstructive pulmonary disease. Am J Respir Crit Care Med 1996; 154: 442-447.

105. Gosselink R, Troosters T, Decramer M. Distribution of muscle weakness in patients with stable chronic obstructive pulmonary disease. J Cardiopulm Rehabil 2000; 20: 353-360. 